\title{
Circulating Osteoprotegerin in Chronic Kidney Disease and All-Cause Mortality
}

\author{
Joanna Kamińska ${ }^{1, *}$ \\ Marek Stopiński ${ }^{1, *}$ \\ Krzysztof Mucha (iD) ${ }^{2,3}$ \\ Michał Pac iD ${ }^{2}$ \\ Marek Gołębiowski ${ }^{4}$ \\ Monika A Niewczas ${ }^{5,6}$ \\ Leszek Pączek ${ }^{2,3}$ \\ Bartosz Foroncewicz (iD) ${ }^{2}$ \\ 'Department of Internal Diseases and \\ Dialysis Unit, West Hospital of Saint John \\ Paul II, Grodzisk Mazowiecki, Poland; \\ ${ }^{2}$ Department of Immunology, \\ Transplantology and Internal Diseases, \\ Medical University of Warsaw, Warsaw, \\ Poland; ${ }^{3}$ Institute of Biochemistry and \\ Biophysics, Polish Academy of Sciences, \\ Warsaw, Poland; ${ }^{4}$ Department of Clinical \\ Radiology, Medical University of Warsaw, \\ Warsaw, Poland; ${ }^{5}$ Research Division, \\ Joslin Diabetes Center, Boston, MA, USA; \\ ${ }^{6}$ Department of Medicine, Harvard \\ Medical School, Boston, MA, USA
}

*These authors contributed equally to this work
Background: Chronic kidney disease (CKD) is associated with cardiovascular disease (CKD), mineral and bone disorder (CKD-MBD) and high mortality. Bone-related factors such as osteopontin (OPN), osteocalcin (OC), osteoprotegerin (OPG) and fibroblast growth factor 23 (FGF23) were linked to cardiovascular complications of CKD and are expected to have predictive value in CKD patients.

Purpose: The aim of this study was to assess the relationship of OPN, OC, OPG and FGF23 to clinical characteristics and to evaluate their ability to predict mortality in patients with different CKD stages.

Methods: The following study groups were enrolled: subjects with end-stage renal disease (38 ESRD), CKD stages 3 and 4 (19 CKD3-4) and non-CKD controls (19), respectively. Blood was withdrawn once to perform the measurements and cardiac computed tomography was used to evaluate coronary calcium score (CS). Patients were followed for 5 years for the ascertainment of their all-cause mortality.

Results: Serum OPN, OC and OPG concentrations increased significantly along with the progression of renal disease. We found a significant positive correlation among these proteins. Additionally, OPN and OPG were significantly and positively correlated to CS. Serum OPG revealed the strongest correlation to the calcium turnover markers of GFR decline and was significantly associated with an increased risk of death in subjects with CKD3-4 or ESRD (HR 5.8, CI 95\%).

Conclusion: Single measurement of osteoprotegerin is associated with 5-year all-cause mortality in patients with CKD3-4 or ESRD. We suggest assessing its concentration, preferably in combination with calcium score, to stratify mortality risks in CKD patients.

Keywords: calcium score, chronic kidney disease, osteocalcin, osteopontin, osteoprotegerin

\section{Introduction}

Chronic kidney disease (CKD) is associated with a high incidence of cardiovascular disease (CVD) and related mortality. ${ }^{1}$ Another common complication of CKD that may also affect CVD is mineral and bone disorder (CKD-MBD). ${ }^{2}$ CKD-MBD is manifested by one or a combination of: 1) abnormalities of calcium, phosphorus, parathyroid hormone, vitamin D and some bone-related cytokine metabolism, 2) abnormalities in bone turnover, and 3) vascular or other soft-tissue calcification. Decline in glomerular filtration in CKD patients causes vitamin D deficiency, derangements in calcium and phosphate homeostasis, and secondary hyperparathyroidism resulting in bone destruction and vascular calcification. ${ }^{3}$ The latter is associated with cardiovascular morbidity and mortality. ${ }^{4}$ It was recently reported that medial arterial calcification in CKD patients is increased and correlates with
Correspondence: Bartosz Foroncewicz Department of Immunology, Transplantology and Internal Diseases, Medical University of Warsaw, Nowogrodzka 59, Warsaw, 02-006, Poland Tel +48-22-502 I 64l

Fax +48-22-502 2127

Email bartosz.foroncewicz@wum.edu.pl 
circulating CVD markers. ${ }^{5}$ Furthermore, calcification score emerged as a significant predictor of long-term survival in $\mathrm{CKD}$ patients. ${ }^{6} \mathrm{CKD}-\mathrm{MBD}$ is a complex disease that is not completely understood. However, some factors secreted by the osteocytes might play an important role in its pathophysiology. These factors are linked to cardiovascular complications of CKD and are expected to have diagnostic predictive value in patients with $\mathrm{CKD}^{7}$

Bone-related factors such as: osteopontin (OPN), osteocalcin (OC), osteoprotegerin (OPG) and fibroblast growth factor 23 (FGF23) are linked to CVD development in CKD patients. ${ }^{5,8-18}$ Plasma OPN levels are independently associated with the presence and severity of diabetic nephropathy ${ }^{9}$ and were found to be expressed by inflammatory cells such as macrophages and highly induced during inflammatory activation. ${ }^{10,11}$ In combination with OPG, they increase the predictability of cardiovascular outcomes. ${ }^{12}$ Serum OC concentrations are significantly lower in non-dialysis CKD patients than in healthy individuals, and correlate with subclinical atherosclerosis in CKD patients. ${ }^{13}$ OPG indirectly, exerts a suppressive effect on osteoclastogenesis and regulates inflammatory and immune responses. ${ }^{14}$ It was found to be associated with increased risk of death in CKD patients and was proposed a marker of atherosclerosis and ischemic stroke. ${ }^{15-18}$ FGF23 is physiologically involved in renal phosphorus excretion and is associated with increased mortality, left ventricular hypertrophy, endothelial dysfunction and progression of CKD. ${ }^{19,20}$ The diversity in study designs and patient populations precludes definite conclusions as to the diagnostic value of these bone-related factors in CKD population.

The aim of this study was to assess the relationship of OPN, OC, OPG and FGF23 to clinical characteristics and to evaluate whether these biomarkers could predict mortality in patients with different CKD stages at baseline.

\section{Materials and Methods}

\section{Patients and Study Design}

The following study groups were enrolled: subjects with end-stage renal disease (ESRD, $\mathrm{n}=38), \mathrm{CKD}$ stages 3 and $4(\mathrm{CKD} 3-4, \mathrm{n}=19)$ and non-CKD controls $(\mathrm{n}=19)$. Patients were enrolled randomly from the group of patients followed-up in our single center. The inclusion criteria were ESRD or CKD 3 and 4, age >18 years, willingness to participate in the study and ability to sign the informed consent. The exclusion criteria included active infections, malignancies, acute cardiologic conditions such as myocardial infarction or atrial fibrillation and pregnancy. The study groups were unintentionally comparable with respect to sex and age. The clinical and biochemical markers of renal function differed significantly between the groups by study design. Clinical characteristics are summarized in Table 1. Fasting blood was drawn once to perform the measurements, and cardiac computed tomography (CT) was used to evaluate the coronary calcium score (CS). The CT safety was discussed with the patients and appropriate information was provided when informed consent was obtained. Patients were followed prospectively for 5 years to ascertain their all-cause mortality. None of the study patients had to be referred to further invasive diagnostics of coronary artery disease based on the CS results. Fatal events were recorded based on patients' hospital and out-patient medical records. During this 5-year period 16 patients died: 13 from the ESRD group and 3 from the CKD group. Cardiovascular causes of death were recorded in 11 ESRD and in 2 CKD3-4 patients. Mortality data were collected blind to the laboratory and CT results. The study received approval from the Ethical Committee at the Warsaw Regional Medical Chamber (Resolution No 08/10) and all individuals gave informed consent prior to enrollment. This study was conducted in accordance with the Declaration of Helsinki and was a continuation of previous investigation aimed at determination of other biomarkers. $^{21}$

\section{Methods}

In the ESRD group, who were dialyzed 3 times per week, blood was taken before mid-week hemodialysis. Blood samples were centrifuged 10 minutes, at $1800 \mathrm{~g}$ at $+4^{\circ} \mathrm{C}$ and stored in small aliquots at $-70^{\circ} \mathrm{C}$ until analysis (Sarstedt tubes, Numbrecht, Germany). The classical inflammatory markers: C-reactive protein (CRP) or highsensitivity CRP (hsCRP) (if CRP was lower than $5 \mathrm{mg} / \mathrm{l}$ ) and procalcitonin, as well as indices of calcium turnover: total and ionized calcium, phosphorus, vitamin D and parathyroid hormone (PTH) were evaluated. MDRD equation was used to estimate glomerular filtration rate $(\mathrm{eGFR})^{22,23}$

\section{Bone-Related Factors}

All bone-related biomarkers were determined with the use of quantitative antibody-based immunoassays in serum samples subjected to one freeze-thaw cycle. OPN, OC and OPG were measured with Milliplex Human Bone 
Table I Clinical Data at Baseline (Modified From ${ }^{21}$ )

\begin{tabular}{|c|c|c|c|c|}
\hline Clinical Characteristics & $\begin{array}{c}\text { Control Group } \\
n=19\end{array}$ & $\begin{array}{c}\text { CKD3-4 Group } \\
n=19\end{array}$ & $\begin{array}{c}\text { ESRD Group } \\
n=\mathbf{3 8}\end{array}$ & $P$ value \\
\hline Age (years) & $62 \pm 9$ & $65 \pm 15$ & $60 \pm 16$ & 0.45 \\
\hline Male n (\%) & $10(53)$ & $9(47)$ & $21(55)$ & 0.87 \\
\hline \multicolumn{5}{|l|}{ Prevalent } \\
\hline Diabetes n (\%) & $2(11)$ & $7(37)$ & $12(32)$ & 0.14 \\
\hline Hypertension n (\%) & $5(26)$ & $16(84)$ & $22(59)$ & $<0.001 *$ \\
\hline CVD n (\%) & $2(\mathrm{II})$ & 7 (37) & $13(34)$ & 0.12 \\
\hline \multicolumn{5}{|l|}{ Biochemical tests [mean (SD)] } \\
\hline eGFR $\left(\mathrm{mL} / \mathrm{min} / \mathrm{I} .73 \mathrm{~m}^{2}\right)$ & $91.28(17.34)$ & $28.59(11.18)$ & $6.70(1.94)$ & by design \\
\hline CRP (mg/l) & $1.66(1.23)$ & $7.5 \mathrm{I}(\mathrm{II} .68)$ & $12.48(31.84)$ & $<0.00 I^{*}$ \\
\hline hs-CRP (mg/l) & $1.73(1.19)$ & $2.89(3.13)$ & - & 0.15 \\
\hline PCT (ng/mL) & - & $0.12(0.11)$ & I.I3 (2.84) & $<0.00 I^{*}$ \\
\hline $\mathrm{pH}$ & - & $7.33(0.11)$ & $7.37(0.02)$ & $0.007 *$ \\
\hline Total protein $(\mathrm{g} / \mathrm{dl})$ & - & $7.45(0.58)$ & $6.84(0.63)$ & $<0.00 I^{*}$ \\
\hline Albumin $(\mathrm{g} / \mathrm{dl})$ & - & $4.09(0.33)$ & $3.99(0.78)$ & 0.61 \\
\hline lonized calcium (mEq/l) & - & $1.23(0.05)$ & $1.09(0.10)$ & $<0.001^{*}$ \\
\hline Total calcium (mEq/l) & - & $4.61(0.30)$ & $4.28(0.58)$ & $0.02 *$ \\
\hline Phosphorus (mEq/l) & - & $2.17(0.43)$ & $3.80(1.06)$ & $<0.001^{*}$ \\
\hline PTH (pg/mL) & - & 190.53 (209.09) & $539.05(461.46)$ & $0.003 *$ \\
\hline Vitamin D (ng/mL) & $27.25(8.05)$ & $26.84(7.14)$ & - & 0.88 \\
\hline Alkaline phosphatase (U/l) & - & | $29.63(|| 3.24)$ & $96.26(33.75)$ & 0.10 \\
\hline
\end{tabular}

Notes: Conversion factors to SI units are as follows: for CRP and hs-CRP ( $\mu \mathrm{g} / \mathrm{l})$ - 1000; for total protein and albumin (g/l) - I0; for 25-hydroxyvitamin D (nmol/l) - 2.496; for PTH $(\mathrm{pmol} / \mathrm{l})-0.105$; for phosphorus $(\mathrm{mmol} / \mathrm{l})-0.0323$; for total and ionized calcium $(\mathrm{mmol} / \mathrm{l})-0.5$. *Indicates statistical significant $\mathrm{p}<0.05$.

Abbreviations: CKD, chronic kidney disease; CRP, C-reactive protein; CVD, cardiovascular disease; ESRD, end stage renal disease; eGFR, estimated glomerular filtration rate; hs-CRP, high sensitivity C-reactive protein; PCT, procalcitonin; PTH, parathyroid hormone.

Metabolism Panel (HBN1A-51K, Millipore Sigma (formerly Millipore), Billerica, MA, USA) on the Luminex platform, following vendor's protocols. This multiplex particle-enhanced platform incorporates laser-based detection system based on flow cytometry fluidics. FGF23 was assayed with ELISA (EZHFGF23-32K, Millipore Sigma (formerly Millipore), Billerica, MA, USA). Inter-assay coefficient of variation was lower than $18 \%$ in each assay. Samples were balanced by caseness. The optical density (ELISA) or fluorescence intensity (Luminex) was matched with the use of 5-parametric logistic standard curve. ${ }^{24}$ Forty-three percent of the FGF23 measurements were not detectable, which was more frequent in subjects with better preserved renal function. Assay sensitivity for FGF-23 was $9.9 \mathrm{pg} / \mathrm{mL}$. Inter-assay coefficient of variations for these biomarkers was $<12 \%$.

\section{Biochemical Tests}

Basic biochemistry measurements were performed with automatic biochemical analyzers: Cobas Integra 400 plus (Roche Diagnostics, Mannheim, Germany) and Elecsys
2010 Roche. Hs-CRP amount was determined with Roche Diagnostics test; the protein electrophoresis - with Beckman, Appraise Paragon; and blood differential test with Sysmex SF3000 and Sysmex K4500.

\section{Coronary Calcium Score}

A 64-row CT scanner (Aquilion 64, Toshiba Medical Systems, Japan) was used to measure CS. The following protocol parameters of this non-contrast enhanced, electrocardiography-gated CT scan were applied: scan number of $40-52$, slice thickness of $3 \mathrm{~mm}$, tube voltage of $120 \mathrm{kV}$, and tube current of $300 \mathrm{~mA}$. Time of rotation was adjusted to the heart rate. The analysis was performed quantitatively according to the Agatston algorithm using Vitrea 2 workstation V3.9 (Vital Images Inc., USA). ${ }^{25}$ Lesions were detected based on density of at least $130 \mathrm{HU}$. Then, they were colour-marked by the software. Experienced radiologist evaluated coronary calcifications. Lesions were scored 1 to 4 depending on their density. CS was determined as a sum of products of each lesion area and its density index. ${ }^{25}$ The mean CT radiation dose ranged from 
Table 2 Circulating Bone-Related and Imaging Markers at Baseline in the Controls, CKD3-4 and ESRD Groups, Respectively. Biomarker Concentrations are Presented as Median (25th, 75th Percentile) Values. Proportion of Detectable FGF23 Values Was $5 \%$ in Controls, $26 \%$ in the CKD3-4 and $92 \%$ in the ESRD Patients

\begin{tabular}{|c|c|c|c|c|c|}
\hline & $\begin{array}{c}\text { Control Group } \\
n=19\end{array}$ & $\begin{array}{c}\text { CKD3-4 Group } \\
n=19\end{array}$ & $\begin{array}{c}\text { ESRD Group } \\
n=38\end{array}$ & $\begin{array}{c}P \text { value CKD3-4 vs } \\
\text { Control }\end{array}$ & $\begin{array}{c}P \text { value } \text { CKD3-4 vs } \\
\text { ESRD }\end{array}$ \\
\hline \multicolumn{6}{|l|}{ Bone-related markers } \\
\hline Osteopontin (ng/mL) & II $(6,15)$ & $27(22,37)$ & $38(27,49)$ & $<0.001$ & $<0.001$ \\
\hline Osteocalcin (ng/mL) & $8.0(5.8,10.5)$ & $20(15,30)$ & $102(39,188)$ & 0.04 & $<0.001$ \\
\hline Osteoprotegerin $(\mathrm{pg} / \mathrm{mL})$ & $45 I(383,607)$ & $729(477,855)$ & II $46(894,1613)$ & 0.10 & $<0.001$ \\
\hline FGF23 (pg/mL) & - & $-(-, 13)$ & $2044(707,52 \mid 3)$ & 0.05 & $<0.001$ \\
\hline \multicolumn{6}{|l|}{ Imaging markers } \\
\hline Coronary calcium score & NM & $338(67,563)$ & $375(38,1144)$ & NA & 0.74 \\
\hline
\end{tabular}

Abbreviations: CKD, chronic kidney disease; ESRD, end stage renal disease; FGF, fibroblast growth factor; NM, not measured; NA, non applicable.

0.7 to $1.4 \mathrm{mSv}$ which qualifies the study as a low-dose technique. The safety of the examination was discussed with the patients.

\section{Statistical Analysis}

Descriptive characteristics were presented as a mean (standard deviation), a median (25th, 75th percentile) or proportions. Skewness and kurtosis metrics of departures from normality were checked and data were transformed to their base 10 logarithms. Crosssectional biomarker comparisons were done with variance analysis for unbalanced design, where biomarker was considered dependent variable. Correlations were evaluated with Spearman correlation coefficients in the analyses adjusted for multiple comparisons (Bonferroni corrected alpha $=0.0029){ }^{26}$ Cox proportional-hazards models tested biomarkers associations with the prospective outcome expressed as hazard ratios per one tertile change of the monotonic marker distribution (one degree of freedom). Ties in the failure time were expressed by the exact conditional probabilities. Nondetectable FGF23 values in the follow-up study group accounted for less than one-third of the biomarker values allowing us to evaluate the effect of FGF23 per tertile treated as a categorical variable. Relevant clinical covariates were considered in building the final model. Principal component analysis is an attractive data reduction approach for highly correlated data and was used here to examine the correlated circulating biomarker data in the context of the baseline CKD status. $^{27}$ The analysis was conducted with an alpha level set to $\alpha=0.05$ with the use of softwares: SAS v. 9.4, Cary, NC and JMP Pro14.

\section{Results}

Total and ionized calcium were significantly lower, in contrast to phosphorus and PTH, which were significantly higher in ESRD than in CKD3-4 patients (Table 1). Serum concentrations of OPN, OC and OPG increased significantly along with the CKD progression (Table 2). All studied factors were significantly higher in ESRD than in CKD3-4 patients, while OPN and OC were also significantly higher in CKD3-4 group than in controls. Moreover, all bone-related factor concentrations had a significant positive correlation among each other and had an inverse correlation to eGFR (Table 3).

The principal component analysis based on the circulating biomarkers revealed decent level of discrimination between the groups and explained more than a half of the variance (Figure 1). The first principal component featured an eigenvalue of 2.758 , whereas the following components had an eigenvalue below 1.0. Loadings of the first component were quite evenly distributed among 4 biomarkers (Supplemental Table 1).

Additionally, OPN and OPG showed a significant positive correlation to $\mathrm{CS}$. OPN was also found to inversely correlate with total calcium. OC and FGF23 similarly significantly correlated positively to PTH and phosphorus and negatively to ionized calcium. Serum OPG revealed the strongest correlation to the clinical consequences of GFR decline. It correlated significantly with all studied calcium turnover markers, with inflammation markers and with $\mathrm{pH}$, albuminemia and proteinemia (Table 3).

Among studied bone-related markers, OPG was the only to increase significantly the risk of death in subjects with CKD3-4 or ESRD. The effect of OPG per one tertile change in the crude analysis was HR (95\% CI): 5.8 (2.2, 
Table 3 Spearman Correlation Matrix for the Studied Biomarkers and Clinical Characteristics

\begin{tabular}{|c|c|c|c|c|c|}
\hline & Osteopontin & Osteocalcin & Osteoprotegerin & FGF23 & Calcium Score \\
\hline \multicolumn{6}{|l|}{ Bone-related markers } \\
\hline Osteopontin & I & $0.57^{* *}$ & $0.56 * *$ & $0.49 * *$ & $0.32 *$ \\
\hline Osteocalcin & $0.57 * *$ & 1 & $0.55 * *$ & $0.74 * *$ & -0.16 \\
\hline Osteoprotegerin & $0.57 * *$ & $0.55^{* *}$ & 1 & $0.59 * *$ & $0.57 * *$ \\
\hline FGF23 & $0.49 * *$ & $0.74 * *$ & $0.59 * *$ & I & 0.20 \\
\hline \multicolumn{6}{|l|}{ Imaging marker } \\
\hline Calcium score & $0.32^{*}$ & -0.16 & $0.57 * *$ & 0.20 & I \\
\hline \multicolumn{6}{|l|}{$\begin{array}{l}\text { Clinical } \\
\text { characteristics }\end{array}$} \\
\hline age & $0.1482 \mid$ & -0.15 & $0.42 * *$ & -0.14 & $0.62 * *$ \\
\hline eGFR & $-0.6 I^{* *}$ & $-0.78 * *$ & $-0.68 * *$ & $-0.81 * *$ & -0.02 \\
\hline CRP & 0,21 & 0.17882 & $0.33 *$ & 0.20 & 0.19 \\
\hline Procalcitonin & 0,20 & $0.54 * *$ & $0.36^{*}$ & $0.65^{* *}$ & 0.03 \\
\hline $\mathrm{pH}$ & 0,07 & $0.34 *$ & $0.33 *$ & $0.55^{* *}$ & 0.02 \\
\hline Total protein & -0.10 & $-0.27^{*}$ & $-0.38^{*}$ & $-0.35^{*}$ & -0.05 \\
\hline Albumin & 0.01 & 0.22 & $-0.3 I^{*}$ & 0.18 & -0.21 \\
\hline lonized calcium & -0.14 & $-0.43 *$ & $-0.50 * *$ & $-0.49 * *$ & -0.19 \\
\hline Total calcium & $-0.26 *$ & -0.26 & $-0.46 * *$ & -0.16 & $-0.31 *$ \\
\hline Phosphorus & 0.20 & $0.66 * *$ & $0.37 *$ & $0.75^{* *}$ & 0.04 \\
\hline Parathyroid hormone & 0.20 & $0.76 * *$ & $0.26 *$ & $0.69 * *$ & -0.03 \\
\hline Vitamin D & -0.14 & -0.16 & -0.11 & -0.18 & 0.25 \\
\hline
\end{tabular}

Notes: Correlation coefficients are presented (*Indicates coefficients significant at alpha $=0.05$ and **Indicates coefficients significant at Bonferroni corrected alpha $=0.0029$ ). Abbreviations: CRP, C-reactive protein; eGFR, estimated glomerular filtration rate; FGF, fibroblast growth factor.

16.0) (Table 4). It remained significant after adjustment for age, sex, baseline CKD status: HR (95\% CI): 5.1 (1.1, 23.3) and further, after adjustment for either CRP, total protein or phosphorus levels. An adjustment for ionized calcium resulted in borderline significance $(\mathrm{p}=0.058)$. The effect of OPG in the model adjusted for age, sex, baseline CKD status and coronary calcium score was HR (95\% CI): $4.1(0.8,20.9) ; \mathrm{p}=0.093$. There was no interaction between OPG and the CKD status $(\mathrm{p}=0.57)$.

\section{Discussion}

We found that osteoprotegerin correlated with lower eGFR, calcium score and other bone-related factors, and was associated with increased 5-year all-cause mortality risk in CKD3-4 or ESRD patients. These observations are in line with the previous reports showing OPG to be elevated in non-diabetic ${ }^{28,29}$ and diabetic ${ }^{30-32}$ CKD patients, and to predict renal function decline, cardiovascular events and all-cause mortality. ${ }^{31}$ OPG has been found to be elevated in association with increased 5- and 10-year kidney function deterioration risk, CKD-related hospitalization, and/or deaths in elderly women. ${ }^{33} \mathrm{~A}$ metaanalysis of 10 studies comprising 2120 CKD patients (including 1723 with ESRD) revealed an association of elevated OPG concentrations with an increased risk of cardiovascular death. ${ }^{34}$ Recently, it was also reported that circulating OPG was significantly associated with CKD diagnosis in hypertensive non-diabetic patients, independently from other variables. ${ }^{35}$ Accordingly, elevated serum OPG levels were associated with higher all-cause and cardiovascular 5-year mortality risk, independent of age, CVD, diabetes, and inflammatory markers, in patients with CKD stages $3-5 .{ }^{36}$ Most of these studies were performed in diabetic patients, and suggest OPG to be a biomarker of CKD progression. ${ }^{37,38}$ Our findings were confirmed on mixed population of diabetic and non-diabetic subjects at different CKD stages, therefore bring novel arguments as to the use of OPG as a mortality predicting marker in CKD patients.

Of note, we found patient age to be positively correlated with OPG, but not with other bone-related markers. This is an important finding in the context of a crosssectional study by Vik et al, who found that OPG variably correlates to eGFR depending on age and renal function. A reverse correlation was found in individuals older than the median age with reduced renal function, whereas a positive association could be observed in younger subjects with normal eGFR. ${ }^{39}$ The mean overall age in this 


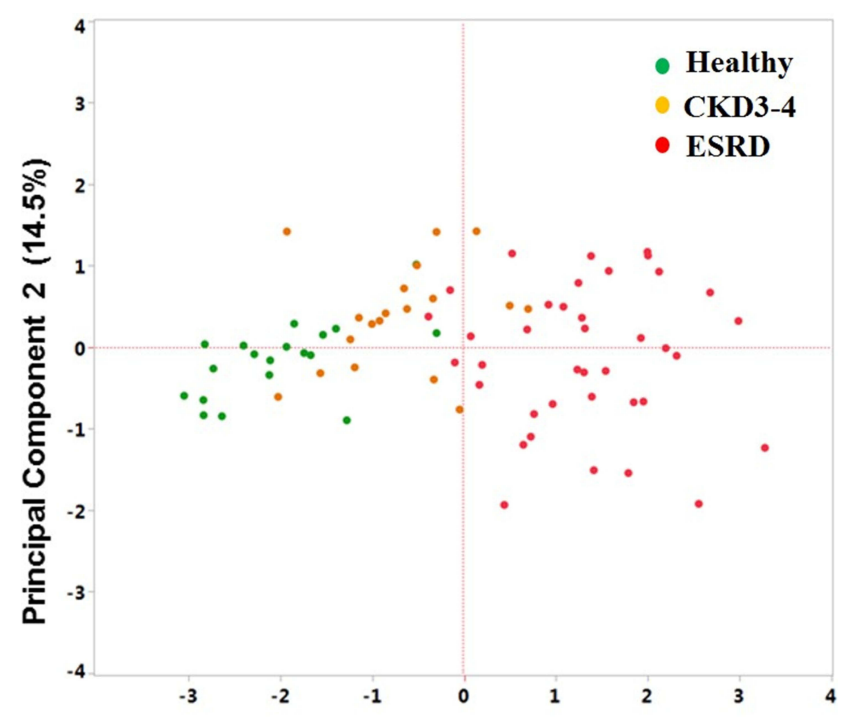

Principal Component 1 (66.9\%)

Figure I Score plot of principal components I and 2 based on the four circulating bone-related biomarkers different between study groups. Each mark represents a study subject. Subjects with ESRD are marked as red circles, those with CKD3-4 are marked as orange circles and the control group is marked with green circles. Description of the $x$ and $y$ axes includes the number of the principal component and the explained variance.

study was 61 years and was comparable to our study groups. This indicated that younger subjects with elevated OPG who develop CKD might have a worse prognosis, particularly because it was also positively correlated with the calcium score.

Analogically to OPG, we found OPN a significant positive correlation with declining eGFR, other bone-related factors and CS. It is known that OPN is produced by the vasculature and bone, is engaged in atherosclerotic plaque formation, and causes renal damage in animal models. ${ }^{40,41}$ In humans, OPN levels may indicate atherosclerosis by means of plaque growth and its rupture susceptibility. Moreover, statins treatment and bypass surgery could reduce OPN concentration. ${ }^{42}$ Therefore, it is not surprising that OPN is linked to the mortality prediction in CKD patients. ${ }^{43}$ Of note, these associations disappeared after adjustment for markers of inflammation. For this reason, combinations of OPN with other biomarkers are sought. Our findings of positive correlations of OPN with OPG and CS are consistent with the results of the NEFRONA Study subanalysis, where the atherosclerotic plaques were assessed in 1043 patients with renal failure in relation to OPG, OPN and sTWEAK concentrations. It was found that elevated OPG or OPN along with the inferior sTWEAK levels significantly correlated to a higher risk of cardiovascular events. Moreover, it was reported that combination of the mentioned biomarkers improved cardiovascular event prognostication in patients with $\mathrm{CKD} .^{12}$

Interestingly, FGF23 was below the level of detection in controls and in the CKD3-4 group. Relation of serum markers to bone expression of specific proteins could partially explain this. In a recent study of patients with different CKD stages and controls, several bone remodeling markers were determined in serum and bone biopsy. This study revealed that sclerostin and PTHR1 were elevated in the earlier CKD stages, whereas FGF23 and phosphorylated b-catenin expression were higher in the advanced CKD. Moreover, significant correlations between serum and bone FGF-23 were established. ${ }^{44}$ One of

Table 4 Cox Proportional Hazard Models for Incident All-Cause Mortality in CKD3-4 or ESRD Patients at Baseline. Crude Models are Presented. Effects of All Biomarkers are Presented per One Tertile Change of a Monotonic Biomarker Distribution, Except for FGF23, Where the Effects are Presented per One Tertile as a Categorical Variable, Respectively

\begin{tabular}{|c|c|c|c|c|c|c|}
\hline \multirow[t]{2}{*}{ Marker } & \multicolumn{3}{|c|}{ Cox Analysis (CKD3-4 + ESRD) } & \multicolumn{3}{|c|}{ Cox Analysis (ESRD Only) } \\
\hline & HR & $95 \% \mathrm{Cl}$ & $P$ value & HR & $95 \% \mathrm{Cl}$ & $P$ value \\
\hline Osteopontin & 1.74 & $0.95-3.18$ & 0.075 & 1.42 & $0.74-2.7$ & 0.29 \\
\hline Osteocalcin & 0.64 & $0.34-1.22$ & 0.17 & 0.61 & $0.32-1.19$ & 0.15 \\
\hline Osteoprotegerin & 5.81 & $2.17-15.56$ & $<0.001$ & 4.87 & $1.74-13.56$ & 0.003 \\
\hline FGF23 TI (ref) & 1.00 & & & 1.00 & & \\
\hline T2 vs TI & 1.99 & $0.30-13.28$ & 0.48 & 1.38 & $0.31-6.15$ & 0.68 \\
\hline T3 vs TI & 1.71 & $0.21-13.37$ & 0.61 & 1.64 & $0.37-7.33$ & 0.52 \\
\hline $\begin{array}{l}\text { Coronary calcium } \\
\text { score }\end{array}$ & 4.65 & $2.14-10.1$ & 0.0001 & 4.09 & $1.88-8.9$ & 0.0004 \\
\hline
\end{tabular}

Note: Bold formatting indicates statistical significance $(p<0.05)$.

Abbreviations: CKD, chronic kidney disease; ESRD, end stage renal disease; FGF, fibroblast growth factor; HR, hazard ratio; T, tertile. 
the study limitations was that our hypothesis-driven approach only focused on four biologically related biomarkers. Future untargeted studies of circulating biomarkers using emerging proteomics technologies will allow us to elucidate these biomarker relationships in greater detail. ${ }^{45}$ Another limitation of our study is the small sample size and patient heterogeneity, which make this study prone to several types of biases. Despite the fact that we have employed a number of careful biostatistical strategies, an analysis adjusted for confounding factors is limited.

\section{Conclusion}

Serum osteoprotegerin is associated with an incident 5-year all-cause mortality in patients with CKD3-4 or ESRD. We suggest assessing its concentration, preferably in combination with calcium score, to stratify mortality risks in CKD patients.

\section{Disclosure}

Dr Joanna Kamińska report personal fees from Roche Poland, outside the submitted work; Dr Bartosz Foroncewicz report congress fee + travel from Astellas and Chiesi, outside the submitted work; Dr Krzysztof Mucha report congress fee + travel from Astellas, outside the submitted work; In addition, Dr Bartosz Foroncewicz, Dr Krzysztof Mucha and Dr Leszek Pączek have patents WO2018141975A1 and WO2017212463 pending. The authors report no other conflicts of interest in this work.

\section{References}

1. Foley RN, Parfrey PS, Sarnak MJ. Epidemiology of cardiovascular disease in chronic renal disease. J Am Soc Nephrol. 1998;9(12 Suppl): S16-S23.

2. Moschella C. Chronic kidney disease-mineral and bone disorder: guidelines for diagnosis, treatment, and management. JAAPA. 2016;29(7):21-29. doi:10.1097/01.JAA.0000484297.49157.9b

3. Yuen NK, Ananthakrishnan S, Campbell MJ. Hyperparathyroidism of renal disease. Perm J. 2016;20:78-83.

4. Noce A, Canale MP, Capria A, et al. Coronary artery calcifications predict long term cardiovascular events in nondiabetic Caucasian hemodialysis patients. Aging (Albany NY). 2015;7(4):269-279. doi:10.18632/aging. 100740

5. Krzanowski M, Krzanowska K, Gajda M, et al. Asymmetric dimethylarginine as a useful risk marker of radial artery calcification in patients with advanced kidney disease. Pol Arch Intern Med. 2018;128(3):157-165. doi:10.20452/pamw.4201

6. Dimkovic N, Schlieper G, Jankovic A, et al. Prognostic value of cardiovascular calcifications in hemodialysis patients: a longitudinal study. Int Urol Nephrol. 2018;50(5):939-946. doi:10.1007/s11255018-1821-1

7. Krzanowski M, Krzanowska K, Gajda M, et al. Pentraxin 3 as a new indicator of cardiovascular-related death in patients with advanced chronic kidney disease. Pol Arch Intern Med. 2017;127(3):170-177. doi:10.20452/pamw.3944
8. Kamińska J, Stopiński M, Krata N, et al. Cardiovascular biomarkers in chronic kidney disease. Forum Nefrol. 2017;10:1-9.

9. Yan X, Sano M, Lu L, et al. Plasma concentrations of osteopontin, but not thrombin-cleaved osteopontin, are associated with the presence and severity of nephropathy and coronary artery disease in patients with type 2 diabetes mellitus. Cardiovasc Diabetol. 2010;9 (1):70. doi:10.1186/1475-2840-9-70

10. Lund SA, Wilson CL, Raines EW, et al. Osteopontin mediates macrophage chemotaxis via $\alpha 4$ and $\alpha 9$ integrins and survival via the $\alpha 4$ integrin. $J$ Cell Biochem. 2013;114(5):1194-1202. doi: $10.1002 /$ jcb. 24462

11. Kahles F, Findeisen HM, Bruemmer D, et al. Osteopontin: a novel regulator at the cross roads of inflammation, obesity and diabetes. Mol Metab. 2014;3(4):384-393. doi:10.1016/j.molmet.2014.03.004

12. Bozic M, Méndez-Barbero N, Gutiérrez-Muñoz C, et al. Combination of biomarkers of vascular calcification and STWEAK to predict cardiovascular events in chronic kidney disease. Atherosclerosis. 2018;270:13-20. doi:10.1016/j.atherosclerosis.2018.01.011

13. Zhang M, Ni Z, Zhou W, et al. Undercarboxylated osteocalcin as a biomarker of subclinical atherosclerosis in non-dialysis patients with chronic kidney disease. J Biomed Sci. 2015;22(1):75. doi:10.1186/s12929-015-0183-6

14. Maruyama K, Takada Y, Ray N, et al. Receptor activator of NF-kappa B ligand and osteoprotegerin regulate proinflammatory cytokine production in mice. J Immunol. 2006;177(6):3799-3805. doi:10.4049/ jimmunol.177.6.3799

15. Yilmaz MI, Siriopol D, Saglam M, et al. Osteoprotegerin in chronic kidney disease: associations with vascular damage and cardiovascular events. Calcif Tissue Int. 2016;99(2):121-130. doi:10.1007/s00223016-0136-4

16. Alkaç C, Alkaç B, Akbaş F, et al. Osteoprotegerin as a marker of atherosclerosis in type 1 and type 2 diabetic patients. Turk J Med Sci. 2015;45:1306-1311. doi:10.3906/sag-1408-37

17. Kim CS, Bae EH, Ma SK, et al. Association of serum osteoprotegerin levels with bone loss in chronic kidney disease: insights from the KNOW-CKD study. PLoS One. 2016;11(11):e0166792. doi:10.1371/ journal.pone. 0166792

18. Üstündağ M, Orak M, Güloğlu C, et al. The role of serum osteoprotegerin and S-100 protein levels in patients with acute ischaemic stroke: determination of stroke subtype, severity and mortality. J Int Med Res. 2011;39(3):780-789. doi:10.1177/147323001103900310

19. Lederer E. Regulation of serum phosphate. J Physiol. 2014;592 (18):3985-3995. doi:10.1113/jphysiol.2014.273979

20. Wal P, Wolf M. FGF23 in chronic kidney disease. In: Kuro M, editor. Endocrine FGFs and Klothos. NY: Springer; 2012:107-125.

21. Kamińska J, Stopiński M, Mucha K, et al. IL 6 but not TNF is linked to coronary artery calcification in patients with chronic kidney disease. Cytokine. 2019;120:9-14. doi:10.1016/j.cyto.2019.04.002

22. Tarantini L, Barbati G, Cioffi G, et al. Clinical implications of the CKD epidemiology collaboration (CKD-EPI) equation compared with the modification of diet in renal disease (MDRD) study equation for the estimation of renal dysfunction in patients with cardiovascular disease. Intern Emerg Med. 2015;10(8):955-963. doi:10.1007/ s11739-015-1260-2

23. van Rijn MHC, Metzger M, Flamant M, et al. Performance of creatinine-based equations for estimating glomerular filtration rate changes over time. Nephrol Dial Transplant. 2020;35(5):819-827. doi:10.1093/ndt/gfy278

24. Gottschalk PG, Dunn JR. The five-parameter logistic: a characterization and comparison with the four-parameter logistic. Anal Biochem. 2005;343(1):54-65. doi:10.1016/j.ab.2005.04.035

25. Agatston AS, Janowitz WR, Hildner FJ, et al. Quantification of coronary artery calcium using ultrafast computed tomography. $J \mathrm{Am}$ Coll Cardiol. 1990;15(4):827-832. doi:10.1016/0735-1097(90) 90282-T 
26. Agresti A. The effect of category choice on some ordinal measures of association. $J$ Am Statistical Assoc. 1976;71(353):49-51. doi:10.1080/01621459.1976.10481475

27. Carey RN, Wold S, Westgard JO. Principal component analysis: an alternative to "referee" methods in method comparison studies. Anal Chem. 1975;47(11):1824-1829. doi:10.1021/ac60361a037

28. Kazama JJ, Shigematsu T, Yano K, et al. Increased circulating levels of osteoclastogenesis inhibitory factor (osteoprotegerin) in patients with chronic renal failure. Am J Kidney Dis. 2002;39(3):525-532. doi:10.1053/ajkd.2002.31402

29. Upadhyay A, Larson MG, Guo CY, et al. Inflammation, kidney function and albuminuria in the Framingham offspring cohort. Nephrol Dial Transplant. 2011;26(3):920-922. doi:10.1093/ndt/ gfq471

30. Rasmussen LM, Tarnow L, Hansen TK, et al. Plasma osteoprotegerin levels are associated with glycaemic status, systolic blood pressure, kidney function and cardiovascular morbidity in type 1 diabetic patients. Eur $J$ Endocrinol. 2006;154(1):75-81. doi:10.1530/ eje. 1.02049

31. Jorsal A, Tarnow L, Flyvbjerg A, et al. Plasma osteoprotegerin levels predict cardiovascular and all-cause mortality and deterioration of kidney function in type 1 diabetic patients with nephropathy. Diabetologia. 2008;51(11):2100-2107. doi:10.1007/s00125-0081123-8

32. Gordin D, Soro-Paavonen A, Thomas MC, et al. Osteoprotegerin is an independent predictor of vascular events in Finnish adults with type 1 diabetes. Diabetes Care. 2013;36(7):1827-1833. doi:10.2337/ dc12-2170

33. Lewis JR, Lim WH, Zhu K, et al. Elevated osteoprotegerin predicts declining renal function in elderly women: a 10-year prospective cohort study. Am J Nephrol. 2014;39(1):66-74. doi:10.1159/ 000357787

34. Huang QX, Li JB, Huang N, Huang XW, Li YL, Huang FX. Elevated osteoprotegerin concentration predicts increased risk of cardiovascular mortality in patients with chronic kidney disease: a systematic review and meta-analysis. Kidney Blood Press Res. 2020;45 (4):565-575. doi:10.1159/000508978

35. Bernardi S, Toffoli B, Bossi F, et al. Circulating osteoprotegerin is associated with chronic kidney disease in hypertensive patients. $B M C$ Nephrol. 2017;18(1):219-227. doi:10.1186/s12882-017-0625-3
36. Marques GL, Hayashi S, Bjällmark A, et al. Osteoprotegerin is a marker of cardiovascular mortality in patients with chronic kidney disease stages 3-5. Sci Rep. 2021;11(1):2473. doi:10.1038/s41598021-82072-z

37. Montanez-Barragan A, Gomez-Barrera I, Sanchez-Nino MD, et al. Osteoprotegerin and kidney disease. J Nephrol. 2014;27(6):607-617. doi:10.1007/s40620-014-0092-x

38. Candido R. The osteoprotegerin/tumor necrosis factor related apoptosis-inducing ligand axis in the kidney. Curr Opin Nephrol Hypertens. 2014;23(1):69-74. doi:10.1097/01. mnh.0000437611.42417.7a

39. Vik A, Brodin EE, Mathiesen EB, et al. Serum osteoprotegerin and renal function in the general population: the Tromsø Study. Clin Kidney J. 2017;10(1):38-44. doi:10.1093/ckj/sfw095

40. Matsui Y, Rittling SR, Okamoto $\mathrm{H}$, et al. Osteopontin deficiency attenuates atherosclerosis in female apolipoprotein E-deficient mice. Arterioscler Thromb Vasc Biol. 2003;23(6):1029-1034. doi:10.1161/ 01.ATV.0000074878.29805.D0

41. Pei Z, Okura T, Nagao T, et al. Osteopontin deficiency reduces kidney damage from hypercholesterolemia in Apolipoprotein E-deficient mice. Sci Rep. 2016;6(1):28882. doi:10.1038/srep28882

42. Wolak T. Osteopontin - a multi-modal marker and mediator in atherosclerotic vascular disease. Atherosclerosis. 2014;236(2):327-337. doi:10.1016/j.atherosclerosis.2014.07.004

43. Barreto DV, Lenglet A, Liabeuf S, et al. Prognostic implication of plasma osteopontin levels in patients with chronic kidney disease. Nephron Clin Pract. 2011;117(4):c363-c372. doi:10.1159/ 000321520

44. Graciolli FG, Neves KR, Barreto F, et al. The complexity of chronic kidney disease-mineral and bone disorder across stages of chronic kidney disease. Kidney Int. 2017;91(6):1436-1446. doi:10.1016/j. kint.2016.12.029

45. Niewczas MA, Pavkov ME, Skupien J, et al. A signature of circulating inflammatory proteins and development of end-stage renal disease in diabetes. Nat Med. 2019;25(5):805-813. doi:10.1038/s41591019-0415-5
International Journal of General Medicine

\section{Publish your work in this journal}

The International Journal of General Medicine is an international, peer-reviewed open-access journal that focuses on general and internal medicine, pathogenesis, epidemiology, diagnosis, monitoring and treatment protocols. The journal is characterized by the rapid reporting of reviews, original research and clinical studies

\section{Dovepress}

across all disease areas. The manuscript management system is completely online and includes a very quick and fair peer-review system, which is all easy to use. Visit http://www.dovepress.com/ testimonials.php to read real quotes from published authors. 